\title{
New Analytical Approximations with Error Estimates for the One-loop Soliton Solution to the Vakhnenko Equation
}

\author{
Yupeng QIN ${ }^{1, a}$, Li ZOU ${ }^{2,3, b,{ }^{*}}$, Zhen WANG ${ }^{1, c}$ and Mingfeng HE ${ }^{1, d}$ \\ ${ }^{1}$ School of Mathematical Sciences, Dalian University of Technology, Dalian, 116024, China \\ ${ }^{2}$ School School of Naval, Architecture State Key Laboratory of Structural Analysis for Industrial \\ Equipment, Dalian University of Technology, Dalian, 116024, China \\ ${ }^{3}$ Collaborative Innovation Center for Advanced Ship and Deep-Sea Exploration, Shanghai, China

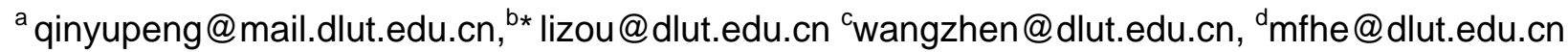

Keywords: Vakhnenko Equation; New Analytical Approximations; Error Estimate; Accuracy; Convergence Rate; Piecewise Perturbation Method

\begin{abstract}
In this paper, based on the traditional homotopy analysis method, we have successfully proposed a new analytical method, namely the piecewise perturbation method (PPM), for solving nonlinear problems. Here we introduce the idea of Newton iteration into the traditional homotopy analysis method to revise the initial guesses, which significantly increases the accuracy and the convergence rate of the series solutions. Further, we apply this method to obtain the one-loop soliton solution of the Vakhnenko equation in order to verify its potential and validity in solving nonlinear problems. With the aid of the optimal value of the convergence-control parameter determined by the averaged residual error technique, comparisons are made between the proposed method and the traditional homotopy analysis method. The results reveal that these new approximations with error estimates possess better accuracy and higher convergence rate than those obtained by the traditional homotopy analysis method.
\end{abstract}

\section{Introduction}

There are few phenomena in various fields of science occurring linearly, most of problems and scientific phenomenon are nonlinear. However, nonlinear problems are much difficult to solve than linear ones, especially by means of analytic methods. In recent decades, some analytical methods were good means of analyzing the nonlinear problems [1-4], and as we know, the homotopy analysis method (HAM) is a famous one for constructing the analytical approximate solutions via power series with the convergence-parameter. This method is superior to the traditional perturbation methods and non-perturbation methods in that it provides us with a convenient way to adjust and control the convergence region and rate of the series solutions. What is more, the HAM has been wider successfully used in science and engineering, see e.g. [5,9,14-18].

Based on the freedom of the initial guess in HAM, our primary aim of this work is to increase the accuracy and convergence rate of the series solutions by revising the initial guesses. In this way, we employ the idea of Newton iteration technique into the traditional homotopy analysis method as follows: first, for a given initial guess of one considered equation, the traditional HAM is used to obtain a fixed finite-order HAM approximation; second, using the idea of Newton iteration, this finite-order HAM approximation is regarded as the new initial guess of the considered equation, and then one can use this new initial guess to obtain the other finite-order HAM approximation, which is the final approximation for this considered equation. In addition, one can determine the optimal value of the convergence-control parameter by the averaged residual error technique suggested by Liao [17]. Throughout this paper we call this proposed method the piecewise perturbation method (PPM), whose essence is the HAM with revised initial guess.

Solitary waves or soliton solutions play an important role in nonlinear field, and to obtain soliton solutions is important in different areas of physics and technology. In recent years, analytical soliton solutions of some nonlinear differential equations have been gotten in various previous articles 
[10-14,16]. In this paper, we will employ the new proposed method to study the Vakhnenko equation [5-8]

$$
\frac{\partial}{\partial x}\left(\frac{\partial}{\partial t}+u \frac{\partial}{\partial x}\right) u+u=0
$$

where $u$ is the dimensionless pressure, $x$ is the spatial variable and $t$ is the time. We should note that the above Vakhnenko equation is a nonlinear equation with loop soliton solutions governing the propagation of high-frequency waves in a relaxing medium.

The paper is arranged as follows. In section 2, we transform the Vakhnenko equation (1) into an ordinary differential equation with boundary conditions. In section 3, a brief review of the traditional HAM and a detail introduction to the proposed method are shown for an abstract nonlinear ordinary differential equation with initial or boundary conditions. In section 4, we apply the new proposed method to analysizing the Vakhnenko equation (1) through the ordinary differential equation obtained in section 2. In section 5, comparisons of the traditional HAM and the proposed method are made to show the validity and superiority of the new method. Discussions and conclusions are provided in section 6.

\section{Normalization of the Vakhnenko Equation}

Using the transformation

$$
x=T+\int_{-\infty}^{X} U(\xi, T) d \xi+x_{0}, \quad t=X, \quad u(x, t)=U(X, T),
$$

Eq. (1) is transformed into

$$
\frac{\partial^{2} U}{\partial T \partial X}+\left(1+\frac{\partial W}{\partial T}\right) U=0
$$

where

$$
W(X, T)=\int_{-\infty}^{X} U(\xi, T) d \xi .
$$

It follows from Eq. (4) that

$$
U(X, T)=\frac{\partial W(X, T)}{\partial X},
$$

and then Eq. (3) becomes

$$
\frac{\partial^{3} W}{\partial T \partial X^{2}}+\frac{\partial W}{\partial X} \frac{\partial W}{\partial T}+\frac{\partial W}{\partial X}=0 .
$$

By applying the traveling wave transformation

$$
W(X, T)=W(\theta), \quad \theta=X-T
$$

into Eq. (6), we obtain the following ordinary differential equation

$$
W^{\prime \prime \prime}(\theta)+\left[W^{\prime}(\theta)\right]^{2}-W^{\prime}(\theta)=0,
$$

where the prime means the derivative with respect to $\theta$. According to the symmetry of $U(X, T)$ in $X$-T space and with the aid of Eq. (5), it is clear that $W^{\prime}(\theta)=W^{\prime}(-\theta)$. Then integrating to both sides of this formula gives $W(\theta)+W(-\theta)=A$, where $A$ is a constant. It is obvious from Eq. (4) that $W(-\infty)$ is equal to zero. From the above discussions, we therefore easily get the following boundary conditions

$$
W(0)=\frac{A}{2}, \quad W^{\prime \prime}(0)=0, \quad W(+\infty)=A .
$$

Finally, under the transformation

$$
W(\theta)=A+\frac{A}{2} g(\theta),
$$

the Eqs. (8) and (9) yield

with the boundary conditions

$$
g^{\prime \prime \prime}(\theta)+\gamma\left[g^{\prime}(\theta)\right]^{2}-g^{\prime}(\theta)=0
$$




$$
g(0)=-1, \quad g^{\prime \prime}(0)=0, \quad g(+\infty)=0,
$$

where $\gamma=A / 2$ is a constant to be determined. The exact value of the parameter $\gamma$ in Eq. (11) is equal to 3 as pointed in [5], which can be also derived from [7]. In the following work, we will analyze the Vakhnenko equation (1) through the ordinary differential equation (11) and its corresponding boundary conditions (12).

\section{Introduction to the Piecewise Perturbation Method}

In this section, to show the new proposed method clearly, we first start with the review of the traditional homotopy analysis method in the subsection 3.1. And then, the basic idea of the new method is given in the subsection 3.2.

\subsection{Review of the traditional homotopy analysis method}

Consider the abstract nonlinear ordinary differential equation

$$
N(g(\theta))=0
$$

with some given initial or boundary conditions, where $N$ is a nonlinear operator, $g(\theta)$ is an unknown function. As Liao pointed in [9], one first construct the zeroth-order deformation equation

$$
(1-q) L\left[\phi(\theta ; q)-g_{0}(\theta)\right]=q h H(\theta) N[\phi(\theta ; q)],
$$

where $q \in[0,1]$ is an embedding parameter, $h \neq 0$ is the convergence-control parameter, $g_{0}(\theta)$ is the initial guess, $H(\theta)$ is an auxiliary function and $L$ is an auxiliary linear operator. It is apparent that when $q=0$ and $q=1$, Eq. (14) becomes $\phi(\theta ; 0)=g_{0}(\theta)$ and $\phi(\theta ; 1)=g(\theta)$ respectively.

Expanding $\phi(\theta ; q)$ in Taylor series with respect to the embedding parameter $q$, we get

$$
\phi(\theta ; q)=g_{0}(\theta)+\sum_{k=1}^{+\infty} g_{k}(\theta) q^{k},
$$

where

$$
g_{k}(\theta)=\left.\frac{1}{k !} \frac{\partial^{k} \phi(\theta ; q)}{\partial q^{k}}\right|_{q=0} .
$$

Supposing that the auxiliary linear operator $L$, the initial guess $g_{0}(\theta)$, the auxiliary function $H(\theta)$ and the convergence-control parameter $h$ are properly chosen such that the series (15) converges at $q=1$, we then obtain the series solution

$$
g(\theta)=\phi(\theta ; 1)=g_{0}(\theta)+\sum_{k=1}^{+\infty} g_{k}(\theta) .
$$

This series solution (17) must be the solution of Eq. (13) by Liao's convergence theorem; see the Chapter 3.3 in [9] for reference.

Differentiating the zeroth-order deformation (14) $k$ times with respect to the parameter $q$, and then setting $q=0$, we have the so called $k$ th-order deformation equation

$$
L\left[g_{k}(\theta)\right]=\chi_{k} L\left[g_{k-1}(\theta)\right]+h H(\theta) R_{k}\left(\vec{g}_{k-1}(\theta)\right), \quad k \geq 1
$$

and its corresponding initial or boundary condition

$$
g_{k}(0)=0
$$

where the vectors and function $\chi_{k}$ satisfying

$$
\begin{aligned}
& \vec{g}_{k}(\theta)=\left\{g_{0}(\theta), g_{1}(\theta), \ldots, g_{k}(\theta)\right\}, \\
& R_{k}\left(\vec{g}_{k-1}(\theta)\right)=\left.\frac{1}{(k-1) !} \frac{\partial^{k-1} N[\phi(\theta ; q)]}{\partial q^{k-1}}\right|_{q=0}
\end{aligned}
$$

and

$$
\chi_{k}= \begin{cases}0, & m=1, \\ 1, & m>1 .\end{cases}
$$

The $k$ th-order deformation equation (18) is linear and therefore $g_{k}(\theta)$ can be easily solved by 
means of symbolic computation software such as MAPLE or MATHEMATICA. Thus, we finally obtain the traditional homotopy analysis solution

$$
g(\theta)=g_{0}(\theta)+\sum_{k=1}^{+\infty} g_{k}(\theta) .
$$

In real applications, we usually use the finite order approximation of Eq. (23), that is, the $r$ th-order HAM approximation

$$
g^{[r]}(\theta)=g_{0}(\theta)+\sum_{k=1}^{r} g_{k}(\theta) .
$$

Here, we point out that the optimal value of the convergence-control parameter $h$ in Eq. (24) could be determined using the so-called averaged residual error technique suggested by Liao [17]. The averaged residual error of the $r$ th-order HAM approximation $g^{[r]}(\theta)$ on the interval $[0, d]$ is defined by

$$
E_{r}=\frac{1}{K} \sum_{j=0}^{K}\left[N\left(g^{[r]}\left(j \frac{d}{K}\right)\right)\right]^{2},
$$

where $d=2$ and $K=20$ are considered for this problem throughout this work, and the optimal value of $h$ is then determined by solving the equation

$$
\frac{\partial}{\partial h} E_{r}=0 \text {. }
$$

Due to Eqs. (25) and (26), the optimal $r$ th-order HAM approximation $g^{[r]}(\theta)$ could be given by substituting the optimal value of $h$ into Eq. (24). We note that, in the next subsection, the work are related to this optimal $r$ th-order HAM approximation.

\subsection{Basic idea of the piecewise perturbation method}

In this subsection, the main idea is to use the optimal $r$ th-order HAM approximations $g^{[r]}(\theta)$ in Eq. (24) to revise the initial guesses.

As mentioned above, we next use the optimal $r$ th-order HAM approximations $g^{[r]}(\theta)$ in Eq. (24) as a new initial guess of Eq. (13). To guarantee the consistency of symbols, we denote the new initial guess by $g_{0}^{[r]}(\theta)$, that is,

$$
g_{0}^{[r]}(\theta)=\text { optimal } g^{[r]}(\theta)
$$

Correspondingly, we have the zeroth-order deformation equation

$$
(1-q) L\left[\phi(\theta ; q)-g_{0}^{[r]}(\theta)\right]=q h H(\theta) N[\phi(\theta ; q)]
$$

the Taylor series

$$
\phi(\theta ; q)=g_{0}^{[r]}(\theta)+\sum_{k=1}^{+\infty} g_{k}^{[r]}(\theta) q^{k}
$$

with

$$
g_{k}^{[r]}(\theta)=\left.\frac{1}{k !} \frac{\partial^{k} \phi(\theta ; q)}{\partial q^{k}}\right|_{q=0},
$$

the $k$ th-order deformation equation

$$
L\left[g_{k}^{[r]}(\theta)\right]=\chi_{k} L\left[g_{k-1}^{[r]}(\theta)\right]+h H(\theta) R_{k}\left(\vec{g}_{k-1}^{[r]}(\theta)\right), \quad k \geq 1
$$

with the initial or boundary condition

where

$$
g_{k}^{[r]}(0)=0,
$$

and

$$
\vec{g}_{k}^{[r]}(\theta)=\left\{g_{0}^{[r]}(\theta), g_{1}^{[r]}(\theta), \ldots, g_{k}^{[r]}(\theta)\right\}
$$




$$
R_{k}\left(\vec{g}_{k-1}^{[r]}(\theta)\right)=\left.\frac{1}{(k-1) !} \frac{\partial^{k-1} N[\phi(\theta ; q)]}{\partial q^{k-1}}\right|_{q=0} .
$$

After solving the $k$ th-order deformation equation (31), a new series solution with the initial guess $g_{0}^{[r]}(\theta)$ of Eq. (13) can be obtained, and has the form

$$
g(\theta)=g_{0}^{[r]}(\theta)+\sum_{k=1}^{+\infty} g_{k}^{[r]}(\theta)
$$

We call this new proposed method the piecewise perturbation method, and correspondingly, the finite order approximation

$$
g^{[m, r]}(\theta)=g_{0}^{[r]}(\theta)+\sum_{k=1}^{m} g_{k}^{[r]}(\theta) .
$$

is called the $[m, r]$ th-order PPM approximation. And via the averaged residual error defined in Eqs. (25) and (26), it is also easy to obtain the optimal [m,r]th-order PPM approximation of Eq. (36) by using $g^{[m, r]}(\theta)$ instead of $g^{[r]}(\theta)$ in Eq. (25).

\section{Application to the Vakhnenko Equation}

In this part, the new proposed method is used to obtain the one-loop soliton solution to the Vakhnenko equation. As examples, we only consider the case of $r=1$ and $r=2$, that is, the [m,1]th-order PPM approximation and the [ $m, 2]$ th-order PPM approximation. We first notice that the traditional HAM has been used for solving this Vakhnenko equation by Wu et al. in [5], from which we can obtain the traditional HAM solution

$$
g(\theta)=g_{0}(\theta)+\sum_{k=1}^{+\infty} g_{k}(\theta),
$$

where

$$
\begin{aligned}
g_{0}(\theta)= & -\frac{4}{3} e^{-\theta}+\frac{1}{3} e^{-2 \theta} \\
g_{1}(\theta)= & \left(\frac{38}{165} h-\frac{4}{3}\right) e^{-\theta}+\left(-\frac{13}{33} h+\frac{1}{3}\right) e^{-2 \theta}+\frac{2}{11} h e^{-3 \theta}-\frac{1}{55} h e^{-4 \theta} \\
g_{2}(\theta)= & \left(\frac{310172}{3493875} h^{2}+\frac{38}{165} h\right) e^{-\theta}-\left(\frac{7573}{99825} h^{2}+\frac{13}{33} h\right) e^{-2 \theta}+\left(-\frac{2701}{33275} h^{2}+\frac{2}{11} h\right) e^{-3 \theta} \\
& +\left(\frac{14303}{166375} h^{2}-\frac{1}{55} h\right) e^{-4 \theta}-\frac{57}{3025} h^{2} e^{-5 \theta}+\frac{24}{21175} h^{2} e^{-6 \theta}, \ldots
\end{aligned}
$$

It should be pointed out that the above results will be directly used in the following computation.

Let us construct the zero-order deformation equation

$$
(1-q) L\left[\phi(\theta ; q)-g_{0}^{[r]}(\theta)\right]=q h H(\theta) N[\phi(\theta ; q), \mathrm{T}(q)],
$$

where the new initial guess $g_{0}^{[r]}(\theta)$ is the optimal $g^{[r]}(\theta)$ determined by Eqs. (37-40), the auxiliary function $H(\theta)$, the linear operator $L$ and the nonlinear operator $N$ are chosen by

$$
\begin{aligned}
& H(\theta)=1, \\
& L=\frac{\partial^{3}}{\partial \theta^{3}}-\frac{\partial}{\partial \theta}, \\
& N[\phi(\theta ; q), \Gamma(q)]=\phi^{\prime \prime \prime}(\theta ; q)+\Gamma(q)\left[\phi^{\prime}(\theta ; q)\right]^{2}-\phi^{\prime}(\theta ; q) .
\end{aligned}
$$

At this moment, the Taylor series of $\phi(\theta ; q)$ and $\Gamma(q)$ with respect to the embedding parameter $q$ yield

$$
\phi(\theta ; q)=g_{0}^{[r]}(\theta)+\sum_{k=1}^{+\infty} g_{k}^{[r]}(\theta) q^{k}, \quad \Gamma(q)=\gamma_{0}+\sum_{k=1}^{+\infty} \gamma_{k} q^{k}
$$


where

$$
g_{k}^{[r]}(\theta)=\left.\frac{1}{k !} \frac{\partial^{k} \phi(\theta ; q)}{\partial q^{k}}\right|_{q=0}, \quad \gamma_{k}=\left.\frac{1}{k !} \frac{\partial^{k} \Gamma(q)}{\partial q^{k}}\right|_{q=0} .
$$

On the other hand, we differentiate the zeroth-order deformation (41) $k$ times with respect to the parameter $q$, and set $q=0$, then the $k$ th-order deformation equation becomes

$$
L\left[g_{k}^{[r]}(\theta)\right]=\chi_{k} L\left[g_{k-1}^{[r]}(\theta)\right]+h R_{k}\left(\vec{g}_{k-1}^{[r]}(\theta), \vec{\gamma}_{k-1}\right), \quad k \geq 1
$$

with the boundary conditions

$$
g_{k}^{[r]}(0)=g_{k}^{[r]{ }^{\prime \prime}}(0)=g_{k}^{[r]}(+\infty)=0,
$$

where

$$
\vec{g}_{k}^{[r]}(\theta)=\left\{g_{0}^{[r]}(\theta), g_{1}^{[r]}(\theta), \ldots, g_{k}^{[r]}(\theta)\right\}, \quad \vec{\gamma}_{k}(\theta)=\left\{\gamma_{0}, \gamma_{1}, \ldots, \gamma_{2}\right\}
$$

and

$$
R_{k}\left(\vec{g}_{k-1}^{[r]}, \vec{\gamma}_{k-1}\right)=\sum_{i=0}^{k-1} \gamma_{k-1-i}\left[\sum_{j=0}^{i} g_{j}^{[r] \prime}(\theta) g_{i-j}^{[r]} '(\theta)\right]+g_{k-1}^{[r]}{ }^{\prime \prime}(\theta)-g_{k-1}^{[r] '}(\theta) .
$$

We demonstrate that Eq. (47) is a linear equation with a general solution in the form

$$
g_{k}^{[r]}(\theta)=g^{*}(\theta)+C_{1} e^{-\theta}+C_{2} e^{\theta}+C_{3},
$$

where $C_{1}, C_{2}$ and $C_{3}$ are the integral constants. It follows from the boundary condition (48) at infinity that $C_{2}$ and $C_{3}$ must be zero. Then the values of $\gamma_{k-1}$ and $C_{1}$ can be determined by the other two boundary conditions in Eq. (48).

By using the Maple to solve Eq. (47) one by one, the specific expressions of $g_{k}^{[r]}(\theta)$ are determined. Further, assuming that $h$ are so properly chosen that the two power series (45) are convergent at $q=1$, we have from (45) that

$$
g(\theta)=g_{0}^{[r]}(\theta)+\sum_{k=1}^{+\infty} g_{k}^{[r]}(\theta), \quad \gamma(q)=\gamma_{0}+\sum_{k=1}^{+\infty} \gamma_{k},
$$

which must be the solution of Eq. (13) [9].

\section{1 [m, 1]-order PPM approximation}

In this subsection, we show the specific representation of the $[m, 1]$-order PPM approximation. Note that approximations throughout this work hold 20 digits, and the calculations are completed by means of the symbolic computation software "Maple".

In the case of $r=1$, the 1th-order HAM approximation $g^{[1]}(\theta)$ of Eq. (13) can be easily given by Eqs. (37-39), and via the averaged residual error formula defined by Eqs. (25) and (26), the optimal value of $h$ in $g^{[1]}(\theta)$ is -1.3312818519920553441 , so the revised initial guess $g_{0}^{[1]}(\theta)$ is determined by the optimal $g^{[1]}(\theta)$ and has the form

$$
\begin{aligned}
g_{0}^{[1]}(\theta)= & -1.6399315780345339580 \mathrm{e}^{-\theta}+.85777769926959755979 \mathrm{e}^{-2 \theta} \\
& -.24205124581673733529 \mathrm{e}^{-3 \theta}+.24205124581673733529 \mathrm{e}^{-4 \theta} .
\end{aligned}
$$

At last, solving the $k$ th-order deformation equation (47) gives the detail formula of the [ $m, 1]$-order PPM approximation

$$
g^{[m, 1]}(\theta)=g_{0}^{[1]}(\theta)+\sum_{k=1}^{m} g_{k}^{[1]}(\theta) .
$$

\section{2 [m, 2]-order PPM approximation}

As to the case of $r=2$, we do the same steps as above to determine the revised initial guess $g_{0}^{[2]}(\theta)$. As a result, the optimal value of $h$ in $g^{[2]}(\theta)$ is equal to -1.3810974496835384214 , which leads to 


$$
\begin{aligned}
g_{0}^{[2]}(\theta)= & -1.8001413034167829735 \mathrm{e}^{-\theta}+1.2767678013935322713 \mathrm{e}^{-2 \theta} \\
& -.65704727327563476830 \mathrm{e}^{-3 \theta}+.2142005302963301093 \mathrm{e}^{-4 \theta} \\
& -.35941659317281095760 \mathrm{e}^{-5 \theta}+.00221619043198364568 \mathrm{e}^{-6 \theta} .
\end{aligned}
$$

In the same way, the $[m, 2]$-order PPM approximation

$$
g^{[m, 2]}(\theta)=g_{0}^{[2]}(\theta)+\sum_{k=1}^{m} g_{k}^{[2]}(\theta)
$$

can be determined by solving the $k$ th-order deformation equation (47) .

\section{Results and Analysis}

Throughout this section, we also analyze the PPM approximations by taking $r=1$ and $r=2$ as examples. We first demonstrate that the exact one-loop soliton solution of the Vakhnenko equation is

$$
u=\frac{3 v}{2} \operatorname{sech}^{2}\left(\frac{\sqrt{v} \zeta}{2}\right), \quad x-v t=3 \sqrt{v} \tanh \left(\frac{\sqrt{v} \zeta}{2}\right)-v \zeta,
$$

where $\zeta$ plays the role of the parameter. From Eqs. (2), (4), (5), (7) and (10), we have

$$
u(x, t)=\gamma g^{\prime}(\theta), \quad x-t=2 \gamma+\gamma g(\theta)+x_{0}-\theta .
$$

At the same time, the symmetry in $x$ - $t$ space implies

$$
x_{0}=-2 \gamma-\gamma g(0)=-\gamma \text {, }
$$

which transform Eq. (58) into

$$
u(x, t)=\gamma g^{\prime}(\theta), \quad x-t=\gamma+\gamma g(\theta)-\theta .
$$

Comparing Eqs. (57) and (60), we get the wave speed $v=1$ and

$$
g(\theta)=\tanh \left(\frac{\theta}{2}\right)-1 .
$$

The following comparisons are relative to this function. We compare the PPM approximation with the traditional HAM approximation by solving the same number of the higher-order deformation equations. Obviously, to get the $r$ th-order HAM approximation $g^{[r]}(\theta)$ and the [m,r]th-order PPM approximation $g^{[m, r]}(\theta)$, the number of the higher-order deformation equations need to be solved are $r$ and $m+r$, respectively. We also note that the following errors are defined by the absolute error, which have the form

$$
E_{P P M}=\left|g_{P P M}-g_{\text {EXACT }}\right|, \quad E_{\text {HAM }}=\left|g_{\text {HAM }}-g_{\text {EXACT }}\right| \text {. }
$$

\subsection{Analysis of the [m, 1]-order PPM approximation}

According to the measure of solving the same number of the higher-order deformation equations, let us use the optimal 4th-order HAM approximation $g^{[4]}(\theta)$ and the optimal $[3,1]$ th-order PPM approximation $g^{[3,1]}(\theta)$ as an example.

Fig. 1 shows the comparison of $g^{[4]}(\theta)$ and $g^{[3,1]}(\theta)$ on the interval [0,3]. They are all agree with the exact solution in Eq. (61) well, this shows these two method are effectiveness in solving this problem. We also found that the curve of the PPM approximation is always below that of the HAM approximation, which means the accuracy and the convergence rate of the PPM approximation are higher than those of the HAM approximation with the aid of the curve of the exact solution. More clear results by estimating the absolute errors of them can be seen in Fig. 2, and it should be demonstrate that the biggest error is below 0.004, which is almost the half of the error of the HAM approximation. Fig. 3 gives the plot of the approximation of $u(x, t)$ in Eq. (60) determined by the optimal [3,1] th-order PPM approximation $g^{[3,1]}(\theta)$, and it is agree well with the exact one-loop soliton solution of Vakhnenko equation in Eq. (57). 


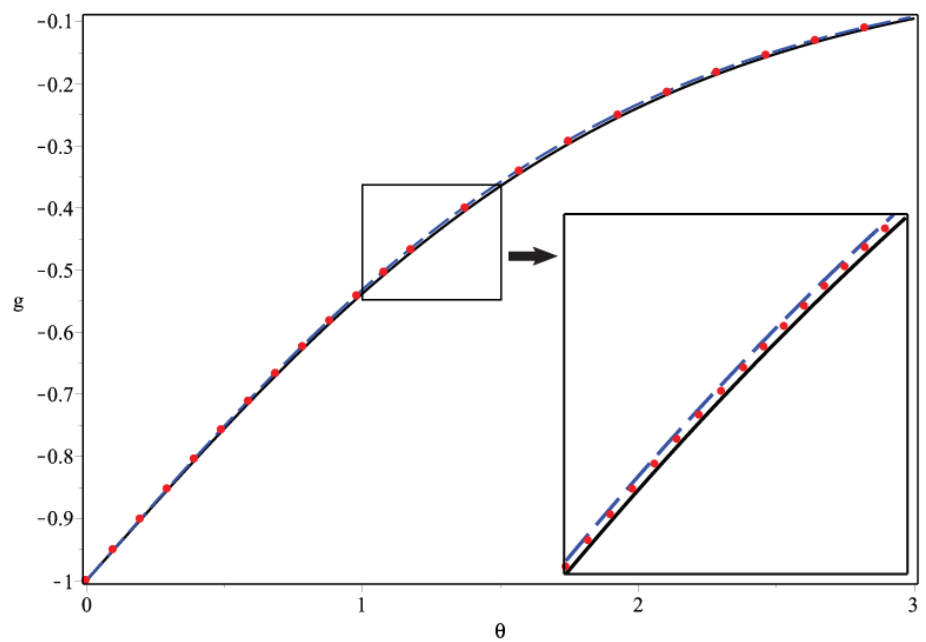

Figure 1. Comparison of the optimal 4th-order HAM approximation $g^{[4]}(\theta)$ and the optimal $[3,1]$ th-order PPM approximation $g^{[3,1]}(\theta)$. Dashed line: 4th-order HAM approximation; solid circle: [3,1]th-order PPM approximation; solid line: exact solution.

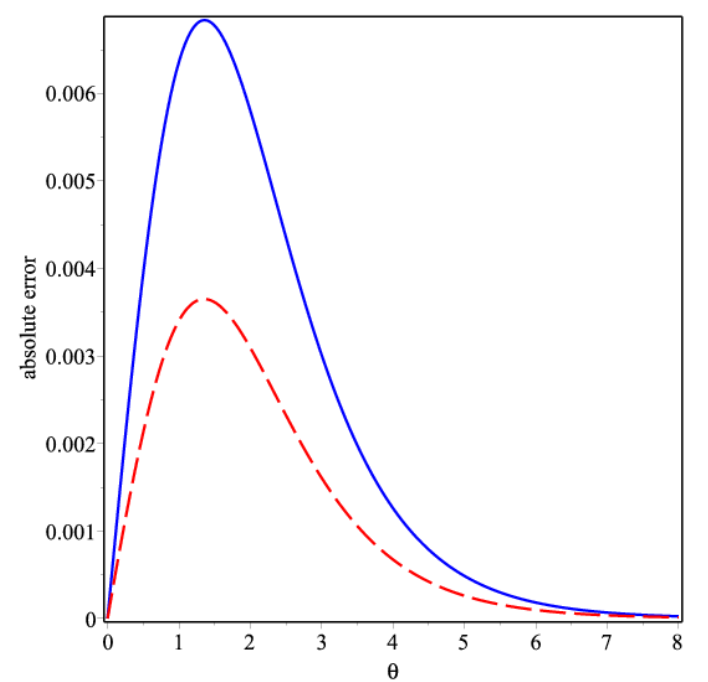

Figure 2. Absolute errors of the optimal 4th-order HAM approximation $g^{[4]}(\theta)$ (shown by solid line) and the optimal $[3,1]$ th-order PPM approximation $g^{[3,1]}(\theta)$ (shown by dashed line).

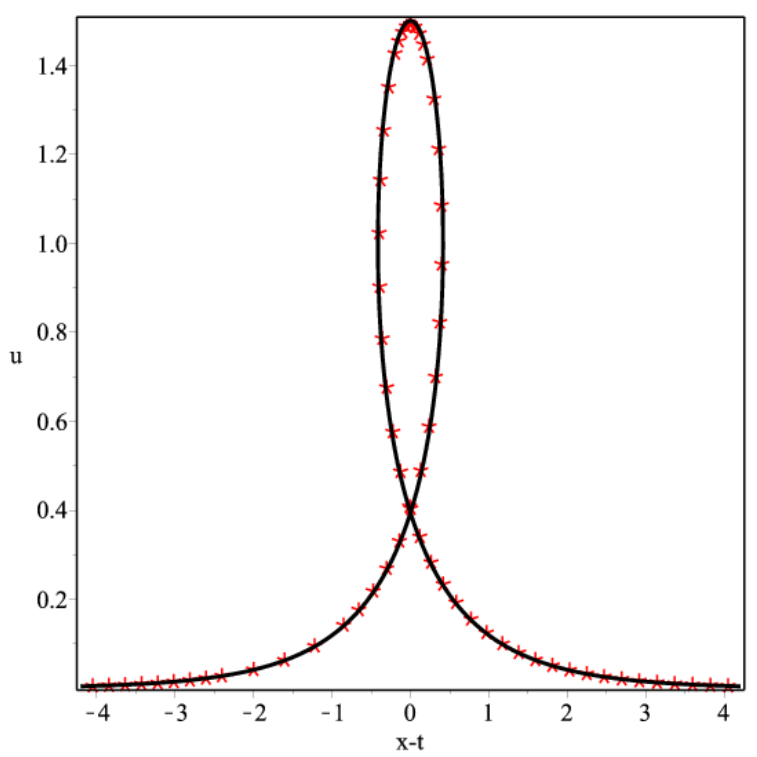

Figure 3. Symbol: The approximation of $u(x, t)$ in Eq. (60) determined by the optimal $[3,1]$ th-order PPM approximation $g^{[3,1]}(\theta)$; solid line: exact solution. 


\subsection{Analysis of the [m, 2]-order PPM approximation}

In this sebsection, we consider the optimal 4th-order HAM approximation $g^{[4]}(\theta)$ and the optimal [2,2]th-order PPM approximation $g^{[2,2]}(\theta)$ to analysis the main results.

The comparison of $g^{[4]}(\theta)$ and $g^{[2,2]}(\theta)$ are given in Fig. 4, and the first finding is that this PPM approximation is also agree with the exact solution in Eq. (61) well. Comparing the two curves in this figure also shows the accuracy and the convergence rate of the PPM approximation are higher than these of the HAM approximation. By considering the absolute errors of them, Fig. 5 shows that the biggest error of this PPM approximation is below 0.003, which is smaller than the half of the error of the HAM approximation. The plot of the approximation of $u(x, t)$ determined by the optimal $[2,2]$ th-order PPM approximation $g^{[2,2]}(\theta)$ are shown in Fig. 6. This approximation is also agree well with the exact one-loop soliton solution of Vakhnenko equation in Eq. (60).

In addition, the comparison of the optimal [3,1]th-order PPM approximation $g^{[3,1]}(\theta)$ and the optimal $[2,2]$ th-order PPM approximation $g^{[2,2]}(\theta)$ are also considered in Fig. 7. It can be seen that the accuracy and the convergence rate of the latter are better than those of the former.

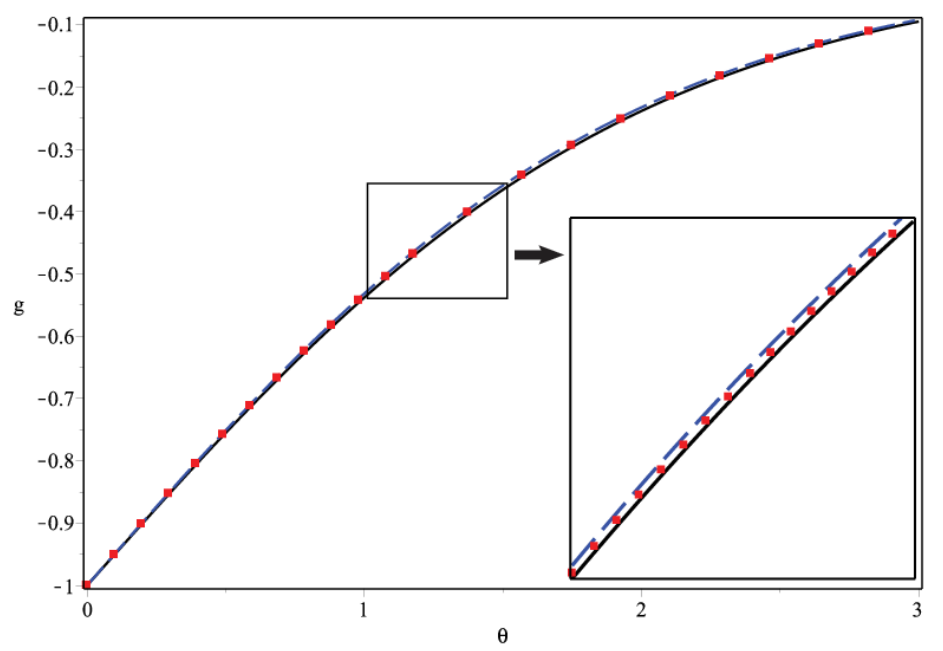

Figure 4. Comparison of the optimal 4th-order HAM approximation $g^{[4]}(\theta)$ and the optimal $[2,2]$ th-order PPM approximation $g^{[2,2]}(\theta)$. Dashed line: 4th-order HAM approximation; solid box: [2,2]th-order PPM approximation; solid line: exact solution.

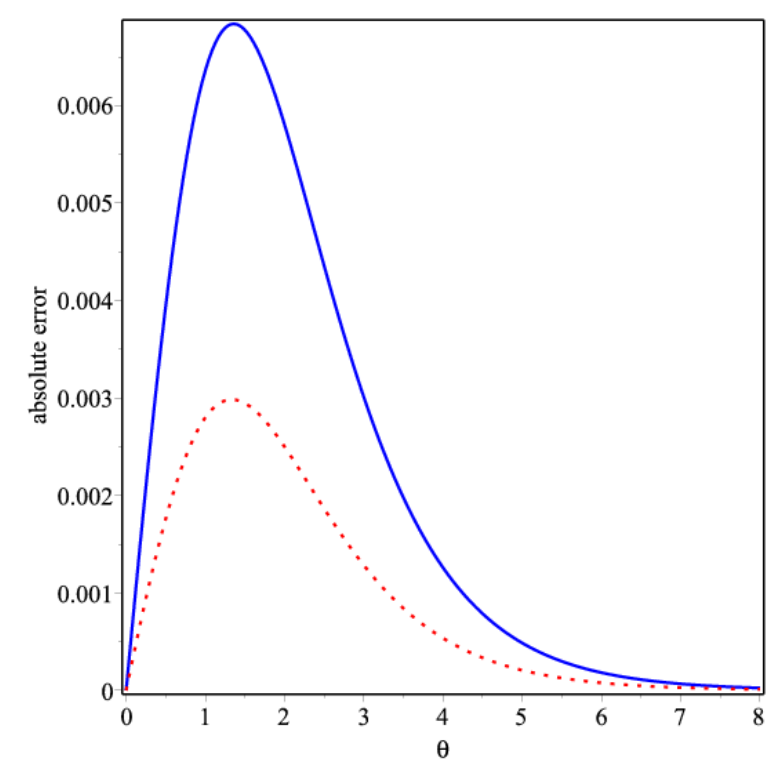

Figure 5. Absolute errors of the optimal 4th-order HAM approximation $g^{[4]}(\theta)$ (shown by solid line) and the optimal [2,2] th-order PPM approximation $g^{[2,2]}(\theta)$ (shown by dotted line). 


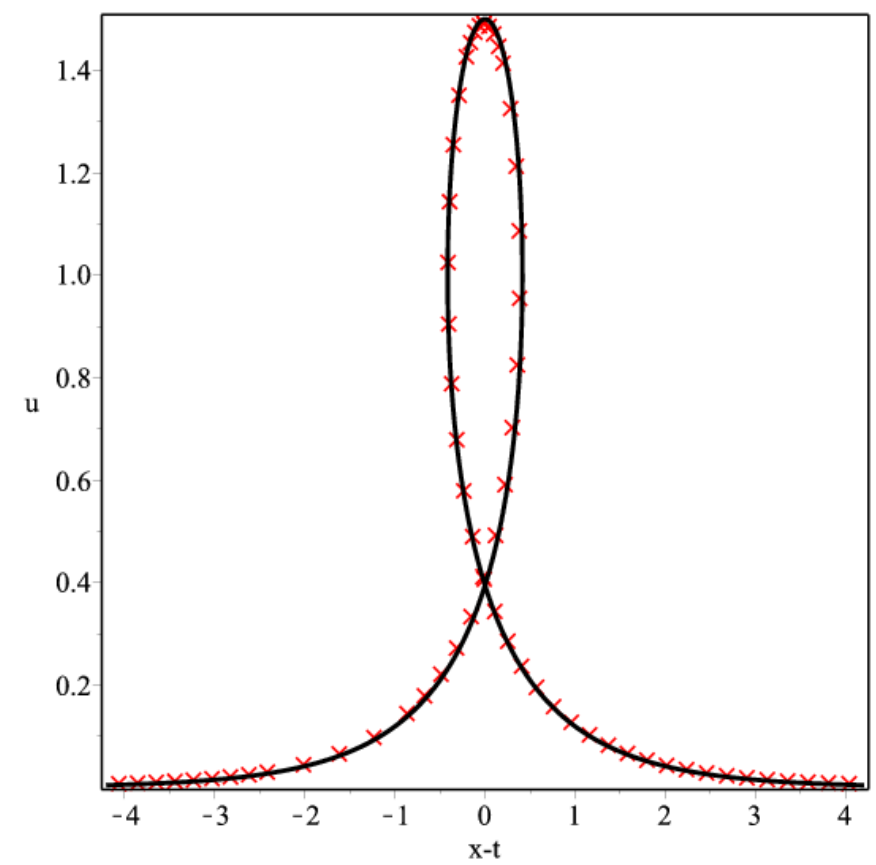

Figure 6. Symbol: The approximation of $u(x, t)$ in Eq. (60) determined by the optimal [2,2]th-order PPM approximation $g^{[2,2]}(\theta)$; solid line: exact solution.

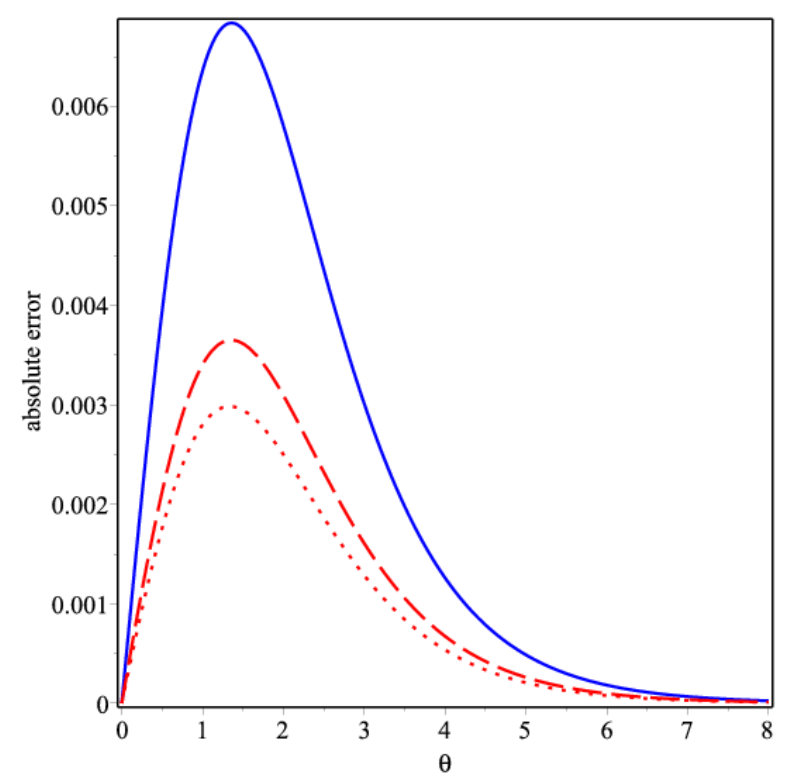

Figure 7. Absolute errors of the optimal 4th-order HAM approximation $g^{[4]}(\theta)$ (shown by solid line) , the optimal $[3,1]$ th-order PPM approximation $g^{[3,1]}(\theta)$ (shown by dashed line) and the optimal $[2,2]$ th-order PPM approximation $g^{[2,2]}(\theta)$ (shown by dotted line).

\section{Conclusion}

In this paper, based on the traditional HAM and the idea of the Newton iteration, a new anlytical method called piecewise perturbation method (PPM) is proposed for solving nonlinear problems. As the application of the proposed method, new approximations for the one-loop soliton solution to the Vakhnenko equation are obtained. The comparisons between the proposed method and the traditional HAM show that these new approximations with error estimates (or the optimal PPM approximations) possess better accuracy and higher convergence rate than those obtained by the traditional HAM. 


\section{Acknowledgement}

This work is partially supported by 973 program (2013CB036101), Natural SciencesFoundation of China under the grant of numbers (51379033, 51522902, 51579040) and the Fundamental Research Funds for the Central Universities (DUT15LK30,DUT15LK43).

\section{References}

[1] E. Yusufoğlu, A. Bekir. The tanh and the sine-cosine methods for exact solutions of the MBBM and the Vakhnenko equations [J]. Chaos Solitons \& Fractals. 2008 (38) 1126-1133.

[2] B.Q. Li, Y.L. Ma, J.Z. Sun. The interaction processes of the N-soliton solutions for an extended generalization of Vakhnenko equation [J]. Applied Mathematics and Computation. 2010 (216) 3522-3535.

[3] V.O. Vakhnenko, E.J. Parkes. The calculation of multi-soliton solutions of the Vakhnenko equation by the inverse scattering method [J]. Chaos Solitons \& Fractals. 2002 (13) 1819-1826.

[4] W.G. Rui, C. Chen, X.S. Yang, Y. Long. Some new soliton-like solutions and periodic wave solutions with loop or without loop to a generalized KdV equation [J]. Applied Mathematics and Computation. 2010 (217) 1666-1677.

[5] Y.Y Wu, C. Wang, S.J. Liao. Solving the one-loop soliton solution of the Vakhnenko equation by means of the homotopy analysis method [J]. Chaos Solitons \& Fractals. 2005 (23) 1733-1740.

[6] A. El-Nahhas. Analytic approximations for the one-loop soliton solution of the Vakhnenko equation [J]. Chaos Solitons \& Fractals. 2009 (40) 2257-2264.

[7] V.O. Vakhnenko, E.J. Parkes. The two loop soliton solution of the Vakhnenko equation [J]. Nonlinearity. 1998 (11) 1457-1464.

[8] A.J. Morrison, E.J. Parkes, V.O. Vakhnenko. The N loop soliton solution of the Vakhnenko equation [J]. Nonlinearity. 1999 (12) 1427-1437.

[9] S.J. Liao. Beyond perturbation: introduction to the homotopy analysis method [M]. BocaRaton: Chapman \& Hall/CRC. 2003.

[10] M.K. Elboree. Derivation of soliton solutions to nonlinear evolution equations using He's variational principle [J]. Applied Mathematical Modelling. 2015 (39) 4196-4201.

[11] F. Azzouzi, H. Triki, P. Grelu. Dipole soliton solution for the homogeneous high-order nonlinear Schrödinger equation with cubic-quintic-septicnon-Kerr terms [J]. Applied Mathematical Modelling. 2015 (39) 1300-1307.

[12] X. Lü, F.H. Lin, F.H. Qi. Analytical study on a two-dimensional Korteweg-de Vries model with bilinear representation, Bäcklund transformation and soliton solutions [J]. Applied Mathematical Modelling. 2015 (39) 3221-3226.

[13] H. Triki, A.M. Wazwaz. On soliton solutions for the Fitzhugh-Nagumo equation with time-dependent coefficients [J]. Applied Mathematical Modelling. 2013 (37) 3821-3828.

[14] L. Zou, Z. Zong, Z. Wang, L. He. Solving the discrete KdV equation with homotopy analysis method [J]. Physics Letters A. 2007 (370) 287-294.

[15] Z. Wang, L. Zou, H.Q. Zhang. Solitary solution of discrete mKdV equation by homotopy analysis method [J]. Communications in Theoretical Physics. 2008 (49) 1373-1378.

[16] Z. Wang, L. Zou, H.Q. Zhang. Applying homotopy analysis method for solving differential difference equation [J]. Physics Letters A. 2007 (369) 77-84. 
[17] S.J. Liao. An optimal homotopy-analysis approach for strongly nonlinear differential equations [J]. Communications in Nonlinear Science and Numerical Simulation. 2010 (15) 2003-2016.

[18] H.Vosoughi, E. Shivanian,S. Abbasbandy. Unique and multiple PHAM series solutions of a class of nonlinear reactive transport model [J]. Numerical Algorithms. 2012 (61) 515-524. 